\title{
The Effect of Immobilization and Sensory Restriction on Children with Phenylketonuria
}

\author{
M.S. Sibinga ${ }^{[33]}$, G.J. Friedman, I.M. Steisel and H.M. Sinnamon \\ Department of Pediatrics, Temple University School of Medicine at St. Christopher's Hospital \\ for Children, Philadelphia, Pennsylvania, USA
}

\begin{abstract}
Extract
The relation between the presence or absence of early immobilization and sensory restriction and current intellectual functioning, language comprehension, and emotional and behavioral patterns was investigated in 30 children with phenylketonuria. The 16 children who had experienced immobilization and sensory restriction, as defined, differed significantly from the other 14 children. In intelligence (WISC) and vocabulary (PPVT) tests, a 20-point difference in Intelligence Quotient was documented between the groups. The incidence of serious behavioral pathology was markedly increased in the children who were subjected to immobilization and sensory restriction during the first three years of life.
\end{abstract}

\section{Speculation}

Practices of medical care commonly employed in pediatric hospitals can substantially affect the future intellectual capacity and behavioral pattern of children with phenylketonuria. Similarly, immobilization and sensory restriction might affect other young pediatric patients unfavorably in the presence of other deleterious influences during hospitalization. One out of four children in pediatric hospitals is subjected to a significant degree of sensory restriction and/or immobilization.

\section{Introduction}

The impact of hospitalization on the emotional and behavioral development of the young patient has been the subject of several recent studies. The potentially harmful after effects have been well recognized [3]. In addition to the physical stress of the illness and its management, the separation of parent and child has been identified as a major potentially traumatic experience. It is thought that the illness may at times be perceived by the child as a punishment, climaxed by parental desertion. Those responsible for the care of children in hospitals have not widely accepted the concept that hospitalization offers an opportunity and a challenge to the physician to assay and support the capacity of the parents, to promote maturity in the child, and to convert his hospitalization to an emotionally growth-promoting experience, rather than to a crippling event engendered by parental doubt and fears [17].

Among the various aspects of hospitalization which may have an unfavorable impact on the child, attention has been specifically drawn to restraint and isolation. The present report identifies preliminary investigations into the potential importance of these frequently encountered conditions.

Three recent monographs $[6,18,21]$ have summarized rapidly developing knowledge concerning the effects of experimental sensory restriction on human behavior and performance. Although children have 
not been used as subjects for such studies, pediatricians might extrapolate some of the principles recently established in this field of research to their own area of concern. Many aspects of the care of infants and children in hospitals have undergone drastic change in the last decades [16]; nevertheless, it appears that some of the manipulatory practices commonly employed by necessity or by convention for therapeutic and diagnostic purposes are very similar to experimental procedures and situational manipulations created for the study of the effects of sensory restriction and motor restraint on the adult human organism. For example, devices to prohibit the moving of arms or legs and an enforced recumbent position to make intravenous infusions of the therapeutic fluids possible not only partially immobilize a child, but also result in drastically altering the sensory proprioceptive input of the child. These procedures have direct parallels in studies of experimental sensory restriction [30]. Other factors which reduce or alter the pattern of stimulation of the child, such as the monotonous interior of a homogeneously lighted oxygen tent or the semi-isolation of a quiet hospital room, also have counterparts in the laboratory study of sensory deprivation [29].

Medical intervention in a hospital setting often has a superficially favorable outcome and may result in minimal interferences with the normal physical growth and development of the child. It has become important to examine the elements of sensory restriction occurring during hospitalization and other medical care undertakings which may impair subsequent mental or emotional function. The stress of sensory restriction on the growing human organism in a hospital setting may have far reaching consequences; these may often be difficult to separate from residual effects of an illness which necessitated the sensory restriction. Increased concern with this potential complication of care is developing in relation to premature infants [7] and is reflected in the shortened duration of postoperative care and convalescent time in pediatric hospitals. Concern for the effects of restraint is by no means new [25]. A recent account of one child's protests and subsequent pathology is given by SENN [19].

The present investigation examines the potentially adverse influences of sensory restriction, physical immobilization and isolation occurring during hospitalization of the child.

Neither the behavioral manifestations nor the reduced intellectual capacity of most patients with phenylketonuria (PKU) is readily explained by current concepts of the pathogenesis of the disease [10, 14]. Reported behavioral aberrations range from mild or moderate neurotic symptoms to psychosis. The incidence of such symptoms is over $90 \%$ [14], and no clear relation with dietary treatment has been established
[15]. Similarly, the variety of behavioral aberrations occasioned by sensory deprivation ranges widely [13]. It appeared worthwhile, therefore, to explore in a group of children with PKU the relation between the presence or absence of early experiences suggestive of sensory restriction and current intellectual functioning, language comprehension, emotional and behavioral patterns, and interpersonal behavior.

\section{Methods}

Thirty children with PKU, part of a larger group of such patients, were studied. They were selected because of the availability of results of extensive psychological testing and clinical assessments and because of geographic accessibility. The diagnosis in all patients was supported by the detection of serum phenylalanine levels one time or another in excess of $40 \mathrm{mg} \%$.

These 30 subjects with PKU were assigned to either a stress (S) or a control (C) group on the basis of interview material obtained from one or both parents, most often from the mother. A detailed inquiry was made to determine whether the child had at any time been immobilized, isolated, or physically restricted. Special attention was devoted to the description of procedures used by the parents or others to control indiscriminate eating, hyperactive or destructive behavior, and such annoying habits as head banging and finger-nail biting. Toward the end of the interview, the parents were asked a number of specific questions to help them recall any instances in the life of the child in which he underwent substantial restriction of physical movement, was isolated, or was deprived of stimulation of any sensory modality.

It was decided arbitrarily to define a stress condition as (1) a period of 24 hours of continuous physical restriction of any part of the body, or (2) a period of three or more days of isolation, confinement, or restriction of stimulation of a given sensory modality, if these conditions had occurred prior to the age of three years.

Prior to the interviews, data were collected on all patients regarding dietary treatment. Dietary effectiveness was judged as optimal, fair, or poor on the basis of reports from the parents, records of levels of phenylalanine in blood, and physical examination of the child.

\section{Intelligence Testing}

Attempts were made to administer the Wechsler Intelligence Scale for Children (WISG) to all subjects over five years of age. The Verbal Scale I.Q. of the WISC was prorated from scaled scores on Information, Comprehension, Similarities and Digit Span subtests. A Performance Scale I.Q. was prorated from scaled 
scores on the Picture Completion, Block Design and Coding subtests. The Full Scale I.Q. was derived according to the standard procedure of combining the Verbal and Performance Scale scores. For the two children less than five years of age (one in each group), the Stanford Binet test (L-M) was substituted for the WISC. In the case of five untestable subjects, the Vineland Social Maturity Scale was administered with the parents serving as informants. Under these conditions, the social quotient was used as a measure of mental development. These tests, as well as subsequent estimates of intellectual and social functioning, were all completed before assignment to the $\mathrm{S}$ or $\mathrm{C}$ groups took place.

\section{Language Comprehension}

Language comprehension was measured by the Peabody Picture Vocabulary Test (PPVT) which was administered and scored according to the directions in the manual. In the PPVT, the child is asked to indicate which of four pictures most closely represents the concept evoked by the stimulus word. If the patient was untestable by this procedure, a PPVT I.Q. was assigned arbitrarily. In two children, speech was absent and a PPVT I.Q. of 25 was scored; for the one child who used sounds to approximate 'mother', 'father', and 'sister', a PPVT I.Q. of 30 was recorded. Two of the children who were not amenable to testing, but who could repeat monosyllabic words, were assigned a PPVT I.Q. of 40.

\section{Diagnostic Impressions}

Diagnostic impressions were obtained by a clinical psychologist on all 30 children during psychological testing. In addition, 22 of the children with PKU were seen by a child psychiatrist. From both psychological testing and psychiatric play interviews, the children were independently classified according to three categories indicating the degree of emotional disturbance: (1) clearly psychotic, (2) questionably psychotic or severely disturbed, and (3) clearly not psychotic.

\section{Interpersonal Behavior}

To assess interpersonal or social behavior, an experimental interaction procedure [22] was performed in a hospital office room, bare of distracting furniture except for a desk and two chairs. A variety of toys, arranged on a shelf placed seven feet from the floor, was intended to stimulate the child to interact with the experimenter in order to obtain these toys. During the first phase of the interaction procedure, the experimenter structured the play situation by removing from the shelf, one at a time, beads, quoits, racing cars, rubber tipped darts, guns, and a target. The experimenter and the child alternated in stringing beads, throwing quoits, and shooting darts at the target. The most competitive activity during the period was that of racing cars the length of the room. This part of the interaction situation, the solicited phase, took about fifteen minutes.

The second phase of the interaction situation was introduced by the experimenter who informed the child that he had some work to do and could no longer play. The experimenter offered the child a toy to play with or, in the event that the child did not make a selection, provided him with a peg board and hammer. Thereafter, he rejected for five minutes the child's interactive efforts. This phase of the interaction situation was intended to reveal how the child responded to this rejection.

The final phase of the interaction situation began when the experimenter informed the child that he was again available for play. During this, the awaited period, however, the experimenter allowed the child to structure the situation. He fulfilled the child's requests by providing whatever toys were requested and by participating in play with the child when invited.

The interaction procedure was observed by two or more judges through a one-way mirror. The child's interactive behavior was rated on several five-point scales which indicate the presence and frequency or absence of various attitudes, including communication, willingness, attention to the experimenter, attention and interest in the toys, and cooperative spirit. The child's social responsiveness was scored by computing an arithmetic average on the scales rated during each phase of the interaction, which permitted evaluation of the child's behavior under different conditions of social stimulus. The total interaction score is the arithmetic average scale rating for the three phases of the procedure [24]. Judges for the interaction were recruited from professional hospital personnel. Interjudge agreement and reliability were measured by Spearman Rank-Order Correlations (rhos). The obtained values were 0.94 for the total score, 0.79 for the solicited, 0.93 for the rejected and 0.71 for the awaited scores. Converting the rhos into $t$ values [19], all were significant at or beyond a p of 0.001 .

Three of the rating scales specifically relevant to the behavior of the patients with PKU, as well as of children who were physically restrained in early childhood [18], were analyzed separately. These rating scales concerned (a) attention to the experimenter, which reflected the child's ability to establish interpersonal relations; (b) attention to toys and objects as an index of object relations; and (c) communicative sounds measuring the child's ability to express his need to play with the toys in the circumstances of the interaction situation. These rating scales were used throughout the different phases of the interaction procedure when appropriate. Rhos 
were calculated as described above and yielded values of 0.80 for attention to the experimenter, 0.81 for attention to toys and objects, and 0.89 for communicative sounds. These values were all significant at or beyond a p of 0.001 [19]. This appeared to establish the reliability of the criterion assessment. To test the significance of the differences between the two groups on the three rating scales, the ratings contributed by two judges were added.

\section{Results}

On the basis of the material collected in the interviews, 16 of the children, 12 boys and 4 girls with a mean age of 9.5 years and a range of 4.1 years to 12.7 years, were placed in the stress (S) group. Fourteen children, 8 boys and 6 girls with a mean age of 9.5 years and a range from 7.1 years to 13 years, were assigned to the control (C) group.

Of the S group, 6 children were currently on a low phenylalanine diet, 6 had formerly been on this diet, and 4 had never had such a diet. Of the total of 12 children who had been on the diet, 10 started it between the ages of 3 and 5 years, and 2 started it before reaching the age of one year. In the control group, 9 of 14 children were currently on the diet, one had formerly been on the diet, and 4 had never been on the diet. Of the total of 10 who had been on the diet, 3 had started the regimen prior to reaching one year of age, and the 7 remaining were put on the diet between the ages of 3 and 5 years. Of the 6 subjects currently on the diet in the $\mathrm{S}$ group, dietary effectiveness was judged to be optimal for 4 and poor for 2 . Of the 9 subjects currently on the diet in the $\mathrm{C}$ group, dietary effectiveness was described as optimal for 4, fair for 3, and poor for 2. Table $I$ presents the ages of the children at the time of diagnosis; these ages coincide with the onset of the dietary regimen for the children who were placed on diets.

The length of time of utilization of the low phenylalanine diet varied from 40 to 112 months in the $S$ group and from 50 to 122 months in the $\mathrm{C}$ group. The

Table I. Ages of subjects at time of diagnosis

\begin{tabular}{lccccccc}
\hline & $0-3$ & $4-6$ & $\begin{array}{c}7-12 \\
\text { months }\end{array}$ & \begin{tabular}{c}
$13-24$ \\
\multicolumn{7}{c}{}
\end{tabular} \\
\hline $\begin{array}{l}\text { Stress } \\
\text { group }\end{array}$ & 0 & 1 & 1 & 3 & 8 & 3 \\
\hline $\begin{array}{l}\text { Control } \\
\text { group }\end{array}$ & 1 & 0 & 2 & 1 & 7 & 3 \\
\hline
\end{tabular}

average duration of dietary treatment was 6 years 3 months for the children in the S group and 6 years 7 months for the children in the $\mathrm{C}$ group.

From interviews with the parents, it was learned that three of the subjects in the control group, compared with fourteen of the subjects in the stress group, had been hospitalized during the first three years of life. Of the three subjects in the $\mathrm{G}$ group who had been hospitalized, the periods of stay were brief, with a minimum of parent-child separation. Of the 16 subjects in the $\mathrm{S}$ group, the most frequent mode of stress was by means of physical restraint. Nine children in the $S$ group had been wholly or partly tied down continuously for a period of three days or longer. One girl was hospitalized for a period of two weeks and on alternate days had been placed in a special apparatus, a Bradford frame, for the purpose of collecting urine samples. Although continuous physical restraint did not meet or exceed the criterion of 24 continuous hours, the child had been subjected to about 150 hours of physical restraint during a two-week period.

Of those children who were physically immobilized during hospitalization, six had been restrained while being treated with intravenous feeding for dehydration associated with upper respiratory infections. Such episodes are known to affect unfavorably the child with PKU. The remaining four children were hospitalized to establish a diagnosis and/or to establish a suitable diet. Three of the children in the $\mathrm{S}$ group were reported to have had fractures or orthopedic difficulties. Two had been in a leg cast for three and four weeks respectively; the third in a hip cast for six months. The remaining three children in the $\mathrm{S}$ group had been subjected to an isolation experience. One of these, a twelveyear-old girl, just under the age of three years at the time, had been hospitalized for a period of 30 days, most of which time was spent in the absence of parents of familiar persons. She remained in a private room and contentedly kept to herself, receiving attention

Table II. Behavior of 30 patients with phenylketonuria as judged by different observers

\begin{tabular}{|c|c|c|c|c|c|}
\hline $\begin{array}{l}\text { एँ } \\
0 \\
0 \\
0 \\
0 \\
0\end{array}$ & 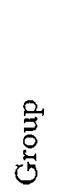 & 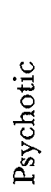 & 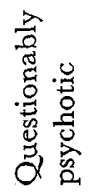 & 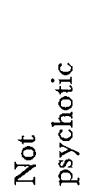 & 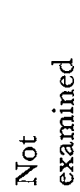 \\
\hline A & $\mathrm{S}$ & 5 & 2 & 9 & 0 \\
\hline B & $\mathrm{S}$ & 5 & 3 & 3 & 5 \\
\hline A & $\mathrm{C}$ & 0 & 0 & 14 & 0 \\
\hline B & C & 0 & 0 & 11 & 3 \\
\hline
\end{tabular}


Table III. Means and standard deviations, and significance of differences between the S and G groups for intelligence, language comprehension and measures of social interaction

\begin{tabular}{lrrrr}
\hline Measure & S group & C group & t & p \\
\hline Intelligence (WISG I.Q.) $_{\text {Language comprehension (PPVT I.Q.) }}$ & $53.69 \pm 23.18$ & $78.93 \pm 16.14$ & 3.57 & $<0.005$ \\
I.S.1 Total $^{2}$ & $2.88 \pm 1.44$ & $3.80 \pm 0.76$ & 2.14 & $<0.005$ \\
I.S. solicited $^{2}$ & $3.14 \pm 1.50$ & $4.40 \pm 0.50$ & 3.15 & $<0.025$ \\
I.S. rejected $_{\text {I.S. awaited }}^{2}$ & $2.64 \pm 0.91$ & $3.02 \pm 1.39$ & 0.78 & \\
$\quad$ Attention to examiner & $2.95 \pm 1.65$ & $4.21 \pm 0.92$ & 2.52 & $<0.025$ \\
$\quad$ Attention to objects & $32.06 \pm 14.48$ & $46.00 \pm 4.95$ & 3.50 & $<0.005$ \\
$\quad$ Communicative sounds & $40.37 \pm 16.81$ & $55.36 \pm 4.80$ & 3.30 & $<0.005$ \\
& $34.06 \pm 19.06$ & $45.07 \pm 12.22$ & 1.84 & $<0.05$ \\
\hline
\end{tabular}

I.S. = Interaction score.

2 Test of significance between means of samples with heterogeneous variances [5].

only for meals and going to the toilet. The remaining cases in the $\mathrm{S}$ group involved two male siblings, the youngest fifteen months and the other 27 months of age, whose mother reported locking both boys in their bedrooms overnight from 6:30 p.m. until 7:30 a.m. for a period of two years. The room was kept empty except for beds and dressers because of the destructiveness of the children. Her intention, the mother reported, was to prevent indiscriminate eating in violation of the diet.

In summary, ten of the 16 children in the S group had experienced physical restraint by being tied down for tests or treatment; three had experienced immobilization by means of casts; and three had been subjected to prolonged isolation or confinement. Of the 14 children in the $\mathrm{C}$ group, three had been briefly hospitalized and none had been subjected to stress as defined.

The behavioral diagnoses of the 30 subjects were determined by independent evaluations of a psychologist (A) and a child psychiatrist (B) who categorized each child as either (1) clearly psychotic, (2) questionably psychotic or severely disturbed, or (3) clearly not psychotic. The results are presented in table II. Combining the first two categories, analysis by chi-square yielded a value of $10.5(\mathrm{p}<0.01)$ for observer $\mathrm{A}$ and $\mathrm{a}$ value of 11.8 ( $p<0.001$ ) for observer $B$. Thus, both psychological and psychiatric evaluations indicated that children in the $\mathrm{S}$ group manifested significantly more severe and frequent symptoms of emotional disturbance than did the patients in the $\mathrm{C}$ group.

The findings regarding intelligence are presented in table III where the mean, standard deviation, and statistical analyses are shown for both groups of patients. A difference of about 20 points in the mean I.Q. between the two groups under consideration is evident. Data on language comprehension and four measures of social interaction are also presented. A difference of 25 points was found between the mean PPVT I.Q.'s of the two groups, while interpersonal behavior was significantly more impaired in the $S$ group with respect to total, solicited and awaited mean scale scores. There was no difference in the response of the children in the two groups during the rejection period.

The children in the $\mathrm{S}$ group displayed significantly greater impairment in interpersonal and object relations and in communicational skills than did the patients in the $\mathrm{C}$ group who had not been subjected to early stress of sensory restriction and isolation.

\section{Discussion and Conclusions}

If it is believed that certain aspects of hospitalization of children with PKU can affect the intellectual and emotional outcome of these patients, it is imperative to scrutinize thoroughly the many variables involved.

While the mental retardation resulting from $\mathrm{PKU}$ can, to an extent, be prevented, many questions regarding the manifestations of this disease remain unanswered. Our ignorance of the pathogenesis of the mental deficiency and the behavioral and other neurological aspects of this disease calls for further elucidation of etiologic factors. The high incidence of this illness (estimates of incidence of heterozygosity are about 1 in 70 to 1 in 50 persons) makes relevant studies urgent.

Clinically, the mental deficiency of untreated patients with PKU is manifested by an I.Q. below 50 in 
$98 \%$ and below 30 in $84 \%$ of the patients [14]. There are several reports indicating that treatment with restriction of dietary intake of phenylalanine results in much more favorable intellectual development. It is generally [12] although not universally [1] believed that early enforcement of such restriction in the first months of life may substantially protect or enhance intellectual development.

The emotional abnormalities of untreated patients with PKU vary from hyperactive and destructive or passive and apathetic behavior to such clear-cut psychotic symptoms as disturbances in thinking, uncontrolled rage and fear reactions, and autistic behavior [23]. It has been said that restlessness, irritability, lethargy, and inability to concentrate disappear with treatment, but this disappearance is independent of intellectual improvement. There is no constant relation between biochemical findings and the degree of mental deficiency and behavioral symptoms in patients with PKU [11]. Institutionalization may lead to regression of intellectual capacity and behavioral adaptation of these patients because of long-term isolation and decreased stimulation [2]. However, more subtle factors in surroundings and in the handling of the affected infant or child may relate significantly to behavioral and intellectual function, especially when such influences occur at vulnerable stages of development and involve children with decreased resistance to such stresses. Restriction of physical movement and restriction or profound alteration of sensory input may form a significant part of current hospitalization practices with children. Since previously reported findings from other groups of children suggest that such stress factors have long-term emotional impact [8], we may ask whether children with PKU exhibit unusual vulnerability in this respect.

Abundant recent experimental data on both animals and man suggest that sensory deprivation and sensory isolation, even of short duration, can profoundly affect psychophysiologic performance [4]. The adaptation patterns reported resemble those seen in PKU and concern activity and arousal patterns, problem-solving behavior, perceptual functioning, and characteristics of thinking and fantasy.

It is important to determine in prospective studies whether one is observing two categories of phenomena with an apparent but spurious relation; viz, (1) conditions of the individual child which led to hospitalization with the stress of restraint and introduction of other environmental changes, and (2) a general level of impairment in intellectual and interpersonal functioning which might reflect primarily greater severity of the disease in the hospitalized group. When variables such as ages of subjects at the time of the study or at the time of diagnosis, as well as incidence, duration, and effectiveness of dietary treatment for the two groups are compared, no obvious differences can be discerned. Review of other details in case material revealed no significant preexisting differences between the groups. It appears unlikely, therefore, that the restraint to which the child in group $\mathrm{S}$ was exposed was the unavoidable consequence of greater severity of PKU.

The severity of the aftereffects of sensory restriction and immobilization is thought to be related to (a) the degree of physical immobilization [26], (b) the number of sensory modalities affected [28], and (c) the knowledge on the part of the subject as to the prospective duration of the sensory restriction [4]. This last factor, in particular, may be of importance in relation to the results of the present study. All children were subjected to the stress prior to the age of 3 years, a time when it is not to be expected that realistic views of the duration of restraint can be imparted to the child.

In sensory restriction experiments, there is a tendency for about $30 \%$ of the volunteer subjects to experience the stress situation as intolerable and to quit the experiment. Recent data [29] indicate that such 'quitters' initially secrete less urinary catecholamines than individuals who can tolerate the procedure for longer periods of time. This is of interest, as some patients with PKU have been reported to be deficient in production of norepinephrine and epinephrine [15]. It seems pertinent to reiterate that certain behavioral characteristics of the majority of children in the $\mathrm{S}$ group, specifically the hyperactivity and passivity patterns [9], resembled the manifestations of psychological disturbances reported previously in patients with PKU and in nonretarded children with the history of physical restraint early in life [8].

The present study involves a relatively small sample of subjects and suffers from some of the weaknesses of retrospective studies. The nature of the present study, however, makes a controlled prospective approach difficult to realize and by necessity the tentative conclusions reached here are deductive rather than inductive in nature. Nevertheless, the data do suggest that in children with PKU, sensory restriction and/or isolation in a hospital during the first three years of life leads to degrees of intellectual retardation and emotional disturbance not experienced by patients with PKU who did not undergo such immobilization or isolation. 
References and Notes

1. Bessman, S.: in: Hyperphenylalanemia without phenylketonuria. Amer. J. Dis. Child. 113: 27 (1967).

2. BJoRnson, J.: Behavior in phenylketonuria. Arch. gen. Psychiat. 10: 89 (1964).

3. Bowlby, J.: Some pathological processes set in train by early mother-child separation. J. ment. Sci. 99: 265 (1953).

4. Cohen, S.J.; Silverman, A.J. and Shmavonian, B. M. : Psychophysiological studies in altered sensory environments. J.psychosom. Res. 6: 259 (1962).

5. Edwards, A.L.: Experimental design in psychological research (Rinehart, New York 1960).

6. Fiske, D.W. and MAdDI, S. R.: Function of varied experience (Dorsey, Homewood, Ill. 1961).

7. Freedman, D.G. and Boverman, H.: The effects of kinesthetic stimulation on certain aspects of development in premature infants. Amer. J. Orthopsychiat. 36: 223 (1966).

8. Friedman, G.J.; Handford, A.H. and Settlage, C.: Child psychologic development: The adverse effects of physical restraint. Paper presented at the meeting of the Regional American Psychiatric Association, Philadelphia, April 1964.

9. Jervis, G. A. : Phenylpyruvic oligophrenia (phenylketonuria). Ass. Res. nerv. Dis. Proc. 33: 259 (1954.)

10. Kennedy, J.L.; Wertelechi, W.; Gates, L.; Sperry, B.P. and Cass, V.M.: The early treatment of phenylketonuria. Amer. J. Dis. Child. 113: 16 (1967).

11. KNox, W.E.: in: The metabolic basis of inherited disease (ed. Stanbury, J.B.; Wyngaarden, J. B. and Fredrickson, D.S.), p.258 (Blakiston-McGraw-Hill, New York 1966).

12. Koch, R.; Acosta, P.; Fischler, K.; Schaeffler, G. and Wohlers, A.: Clinical observations on phenylketonuria. Amer. J. Dis. Child. 113: 6 (1967).

13. Leiderman, P. H.; Mendelson, J.H.; Wexler, D. and Solomon, P.: Sensory deprivation: Clinical aspects. Arch.intern. Med. 101: 389 (1958).

14. Lyman, F.L. (ed.): Phenylketonuria (Thomas, Springfield, Ill. 1963).

15. Nadler, H.G. and Hsia, D.Y.-Y.: Epinephrine metabolism in phenylketonuria. Proc.Soc.exp. Biol., N.Y. 107: 721 (1961).

16. Robertson, J.: Young children in hospitals (Basic Books, New York 1962).

17. Rose, J. and Sonis, M.: The use of separation as a diagnostic measure in the parent-child emotional crisis. Amer.J. Psychiat. 116: 409 (1959).
18. Schultz, D.P.: Sensory restriction, effects on behavior (Academic Press, New York 1965).

19. Senn, M.J.: in: Emotions and symptoms in pediatric practice; Advanc. Pediat. 3: 69 (1948).

20. Sregel, S.: Nonparametric statistics for the behavioral sciences (McGraw-Hill, New York/Toronto/ London 1956).

21. Solomon, P.; Kubzansky, P.E. ; Leiderman, P.H.; Mendelson, J. H.; Trumbull, R. and Wexler, D. (eds.): Sensory restriction (Harvard University Press, Cambridge, Mass. 1961).

22. Steiser, I. M.; Friedman, G.J. and Wood, A. G., Jr.: Interaction patterns in children with phenylketonuria. J. cons. Psychol. 31: 162 (1967).

23. Steisel, I. and Friedman, G.J.: Unpublished observations.

24. Wood, A. G.; Friedman, G.J. and Steisel, I. M.: Psycho-social factors in phenylketonuria. Amer.J. Orthopsychiat. 37: 671 (1967).

25. YARROW, L.J.: in: Review of child development research (ed. Hoffman, M.L. and Hoffman, L.W.), p. 93 (Russell Sage Foundation, New York 1964).

26. Zubek, J.P.: Counteracting effects of physical - exercises performed during prolonged perceptual deprivation. Science 142: 504 (1963).

27. Zubek, J.P.: Urinary excretion of adrenaline and non-adrenaline during prolonged immobilization. J.abnorm. Psychol. 73: 223 (1968).

28. Zubek, J.P.; Sansom, W. and Prysiazniuk, A.: Intellectual changes during prolonged perceptual isolation. Canad.J. Psychol. 14: 233 (1960).

29. Zubek, J. P.; Welch, G. and SAunders, M. : EEG changes during and after 14 days of perceptual deprivation. Science 139: 490 (1963).

30. Zubek, J. P. and Wilgosh, L. : Prolonged immobilization of the body: changes in performance and in the electroencephalogram. Science 140: 306 (1963).

31. This study was supported in part by Research Grants HD 0143-01 and HD 0143-02 from the National Institute of Child Health and Human Development, Grant 416 from the Children's Bureau, United States Department of Health, Education and Welfare, and by one from the Orthopedic Foundation of Philadelphia.

32. The authors are indebted to Drs. J.A. Burland, Gabriel D'Amato, Marg A.Forman, Roger D. Freeman and Paul MaIlhenny for their contributions to the study.

33. Requests for reprints should be addressed to: Dr. MaArten S. Sibinga, St. Christopher's Hospital for Ghildren, 2600 N. Lawrence Street, Philadelphia, Pa. 19133 (USA). 